\title{
The Set-Up and Implementation of Fully Virtualized Lessons with an Automated Workflow Utilizing VMC/Moodle at the Medical University of Graz
}

\author{
http://dx.doi.org/10.3991/ijet.v6i4.1784 \\ Herwig Rehatschek, Gernot Hölzl, Michael Fladischer \\ Medical University Graz, Graz, Austria
}

\begin{abstract}
With start of winter semester 2010/11 the Medical University of Graz (MUG) successfully introduced a new primary learning management system (LMS) Moodle. Moodle currently serves more than 4,300 students from three studies and holds more than 7,500 unique learning objects. With begin of the summer semester 2010 we decided to start a pilot with Moodle and 430 students. For the pilot we migrated the learning content of one module and two optional subjects to Moodle. The evaluation results were extremely promising - more than $92 \%$ of the students wanted immediately Moodle - also Moodle did meet our high expectations in terms of performance and scalability. Within this paper we describe how we defined and set-up a scalable and highly available platform for hosting Moodle and extended it by the functionality for fully automated virtual lessons. We state our experiences and give valuable clues for universities and institutions who want to introduce Moodle in the near future.
\end{abstract}

Keywords-eLearning, Moodle cluster, fully automated virtual lessons

\section{INTRODUCTION AND BACKGROUND}

Since 2002 the Medical University of Graz (MUG) fosters eLearning via its portal Virtual Medical Campus Graz (VMC) [1], [2], [3].

With start of winter semester 2010/11 the MUG successfully introduced a new primary learning management system (LMS) VMC/Moodle, which is now the third generation of LMS - after the LMS VMC 1.0 and Join2Learn - provided by the MUG for its students. VMC/Moodle as the primary LMS of the MUG currently serves more than 4,300 students from three studies which generate in average 100,000 activities a day and holds more than 10,600 unique learning objects.

Within the second LMS Join2Learn we already started to realize a concept for fully automated virtual lessons [4], however, the LMS did not meet our demands in terms of scalability and performance. So we decided at begin of 2010 to introduce a new LMS. After having defined selection criteria we started an evaluation of existing LMS. From this first evaluation Moodle [5] and Olat [6] turned out to be those fitting best to our criteria. Based on the facts that Moodle has the huger online community and promised due to its script implementation to offer a higher performance and easier installation we decided to start a pilot with Moodle and 430 students with begin of the summer semester 2010. For the pilot we migrated the learning content of one module of the diploma study human medicine and two optional subjects to Moodle. At the end we made an evaluation amongst the students. The evaluation results were extremely promising - more than $92 \%$ of the students wanted Moodle immediately - also Moodle did meet our high expectations in terms of performance and scalability. So we decided not to evaluate a second LMS but to introduce Moodle with the start of the winter semester 2010/11 as the main LMS of the MUG.

This decision involved the migration of the entire learning content, the set-up of a hardware platform capable of serving 4300 students and thousands of external users and offering a highly availability, and the adaption of Moodle - referred to from now on as VMC/Moodle - by the implementation of an interface to our central administration system in order to realize our fully automated concept for virtual lessons.

Within this paper we describe how we defined and setup a scalable and highly available platform for hosting VMC/Moodle and extended it by the functionality for fully automated virtual lessons. We state our experiences and give valuable clues for universities and institutions who want to introduce Moodle in the near future.

\section{TECHNICAL REALIZATION}

\section{A. Realization of a highly reliable, available and scalable Moodle platform}

About $9 \%$ of the mandatory curriculum of human medicine are realized in so called "virtual lessons" (see next chapter for a more detailed description) which must be executed by students within a certain timeframe in order to proceed with their study. Due to this fact the MUG has very high demands on the availability of its LMS. Furthermore the LMS must be able to handle up to 7,000 users, 500 in parallel and shall grant response times $<2$ seconds. Next to these basic requirements the LMS has to be scalable to a larger amount of maximum and parallel users.

From the technical point of view there was a must criteria on the operating system which should be SuSE Linux Enterprise Server and a soft criteria on the database where an Oracle database was preferred, since the MUG uses Oracle. 
Based on these general requirements we decided to go for an active / active cluster configuration with two load balancers and a highly redundant storage (SAN - storage area network) as given in Figure 1. The two load balancers distribute requests from the users equally on currently two servers, who share exactly the same database. The system remains stable and fully performant in case one server or one load balancer goes down. Due to the highly redundant storage failure of single hard disks does not result in loss of data nor has it any influence on system availability. In case performance has to be increased further servers can be added to the cluster, which makes the platform scalable.

The set-up of the cluster was executed by selecting the hardware, installation of a pilot system and evaluating it (especially the connection Moodle and Oracle), installation of a production system and testing it, installation of a mirror system for testing Moodle updates before installing them on the production system, and the go-live of the Moodle cluster as the main LMS of the MUG with $1 \mathrm{Oc}-$ tober 2010

\section{B. Extending Moodle in order to execute fully automated virtual lessons}

Within the diploma study human medicine eLearning is an integral part of the mandatory part of the curriculum. Currently about $9 \%$ - this corresponds to 404 lessons á 45 minutes - of this study are fully virtualized, meaning that students can learn this content without having to attend any face-to-face classes. So students have a significant amount of flexibility to organize their study individually and to decide for themselves where and when they want to learn.

These fully virtualized parts are called "virtual lessons" and have to contain at least online studying material and an online quiz a so called web based training (WBT). The purpose of the WBTs is to check, whether the students have reached the learning objectives of this virtual lesson. Even though the LMS VMC/Moodle offers a huge amount of different types of quizzes, only those types are allowed within virtual lessons which can be evaluated by the system fully automatically without any interaction needed from the teachers. Hence currently we allow for WBTs multiple/single choice questions, gap filling questions, numerical questions and matching questions.

As virtual lessons are an integral part of the curriculum virtual lessons must be executed by students within the time the virtual lesson is offered in order to proceed with their study. This is similar to normal seminars and exercises, where students have to fulfil a certain degree of presence in order to get a grade. However in difference to face-to-face lectures virtual lessons provide the flexibility to execute them at any time within the duration the module is offered.

If a teacher wants to virtualize one of his classes one single preparation step has to be performed. Together with the department VMC online learning material and WBTs within the LMS VMC/Moodle have to be authored. After the eLearning content of the virtual lesson is available the title will be synchronized within MEDonline [7], the central lecture and exam administration system for all studies of the MUG. The virtual lesson is now available for being planned. Technically a unique ID is generated for the virtual course within VMC/Moodle, which is transferred together with the title to MEDonline during synchronization. This links the two systems MEDonline and VMC/Moodle logically together.

Even though virtual lessons are specifically tagged within MEDonline, they are treated as all other lectures within the system. Similar to normal lectures also each virtual lesson has a start date. For virtual lessons this is the date, from where the WBT parts of the virtual lessons are automatically generated within VMC/Moodle and presented to all students who are currently enrolled for the module where the virtual lesson is a part of it.

In Figure 2. an example for a virtual lesson within MEDonline and a part of its virtual content within VMC/Moodle is given. In the left bottom a lecture from MEDonline "sv1: Spezielle angiologische Therapieverfahren bei Arteriopathien (Specific therapies for angiological arteriopathies)" is listed, on the right upper side the associated virtual lesson in VMC/Moodle (containing the WBT) is shown to which the lecture in MEDonline is connected via the unique ID. In the MEDonline view also the type "virtuell" (virtual - identifies this lecture as a virtual lecture) and the starting date of the virtual lecture (3.3.2009 - 3 March 2009) can be seen. As explained before this is the date from where the virtual lecture will be presented to students within VMC/Moodle who are enrolled for this virtual lesson.

The end dates for the virtual lessons are not within MEDonline and hence have to be provided manually to VMC/Moodle. However, there exist two general rules from which at least a semi-automated process could be derived and programmed in order to set the end dates by the system, which can then be manually overwritten by the department VMC in case a different end date has to be set. These rules are: virtual lessons which are part of modules, are generally offered over one time slot with a duration of 5 weeks. Virtual lessons which are part of ac-

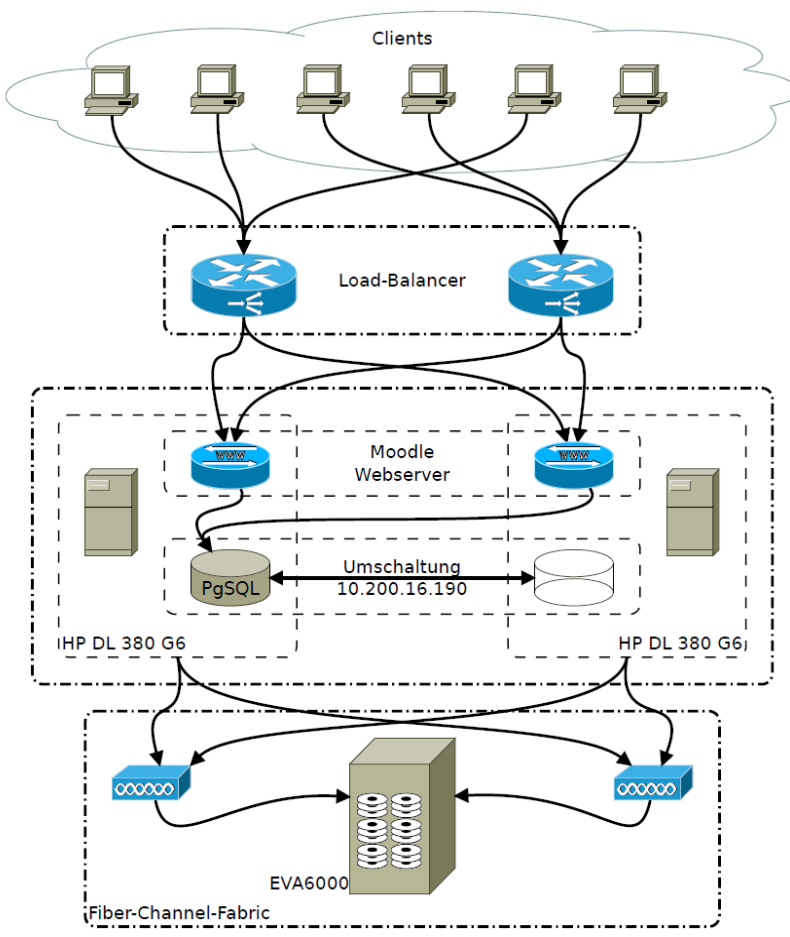

Figure 1. Highly available and scalable Moodle cluster configuration of the MUG 
PAPER

The Set-Up and Implementation of Fully Virtualized Lessons with an Automated Workflow Utilizing VMC/Moodle at THE MEDiCAL UniVERSITY OF GRAZ

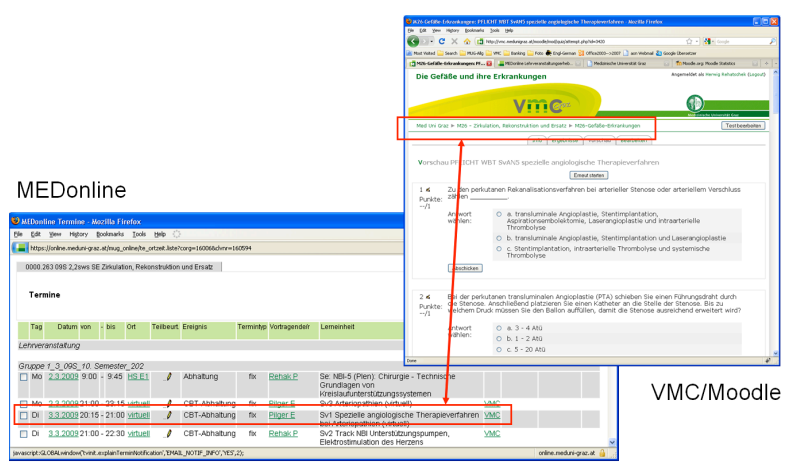

Figure 2. Corresponding virtual lessons in MEDonline and VMC/Moodle

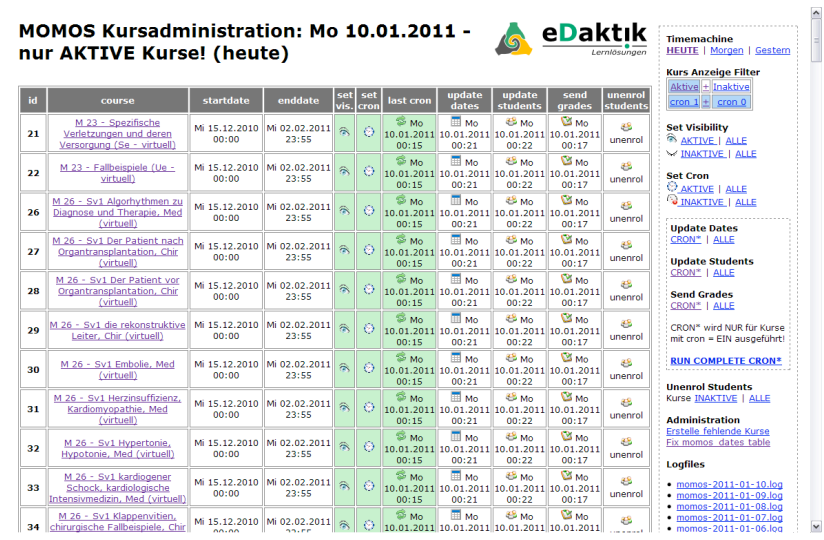

Figure 3. Virtual lesson management interface from VMC/Moodle

companying lectures are offered over one semester. For the management of the virtual lessons an own administration user interface was developed which is given in Figure 3 .

This interface allows employees of the department VMC to comfortably administer the virtual lessons. It gives an overview of all virtual lessons and their start and end dates. Furthermore it allows manually hiding and showing certain virtual lessons, to show the synchronization time with MEDonline, to manually synchronize with MEDonline data and to manually transmit grades of virtual lessons to MEDonline.

When the start date of the virtual lesson is reached, a task is generated for those students who are enrolled for this specific lesson. Students can access these tasks directly from the first page after their login into VMC/Moodle by clicking on a link called "overview on my virtual mandatory tasks". After clicking on this link a dynamic list is generated of all virtual lessons the student is currently enrolled and which he must execute in order to proceed with his study. For each virtual lesson the name, the end date and the number of trials already performed by the student is provided. If the virtual lesson has not been executed so far, a text indicating, that this virtual lesson still must be executed is displayed.

An example of a dynamically generated list with virtual lessons to be executed by a student is given in Figure 4 . Students can now execute the WBTs of the virtual lessons as often as they like until the end of the virtual lesson is reached. The grade is automatically calculated, the best grade reached is stored. Grades are automatically transmitted back to MEDonline every day shortly after mid-

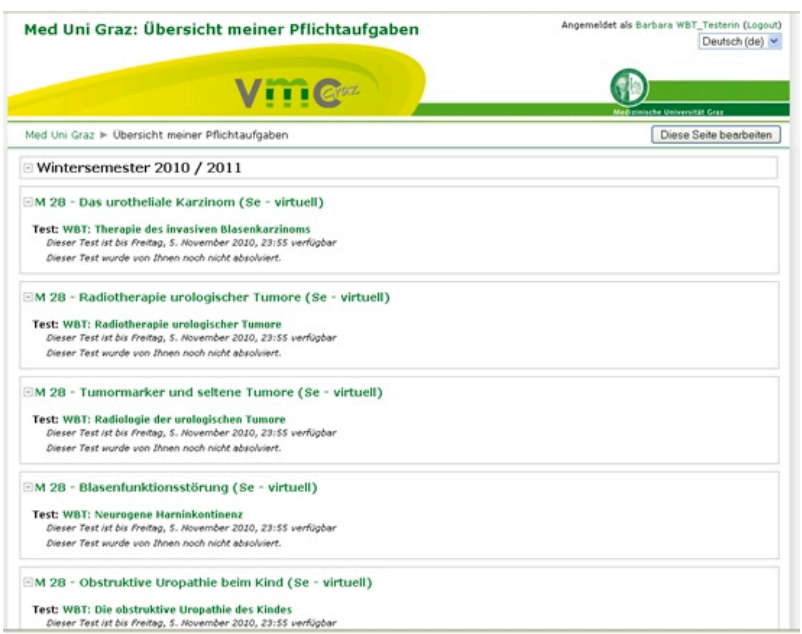

Figure 4. Dynamically generated list of virtual lessons which is displayed to students

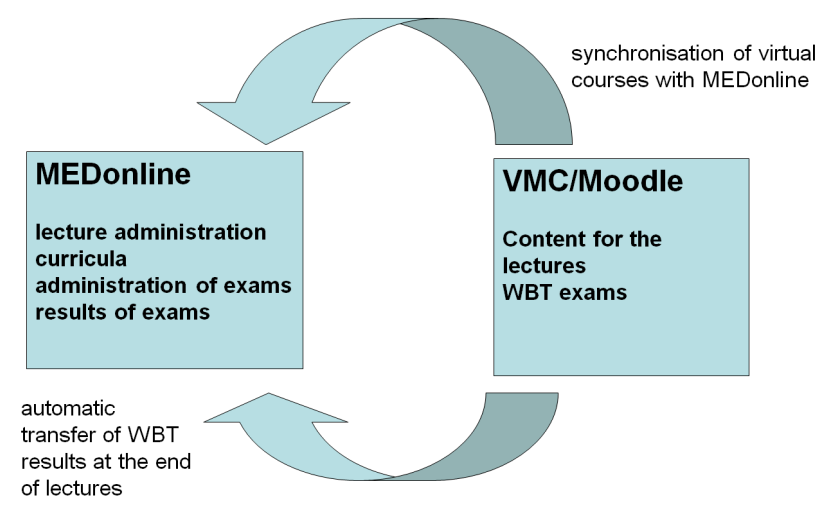

Figure 5. Synchronisation process between MEDonline and VMC/Moodle

night. After the end date the virtual lessons disappear from the student overview page and cannot be executed anymore by them. The grade from the best trial is from now fixed in MEDonline and is from there further executed.

From now on the process is fully automated and starts with the planning of the virtual lectures within the study year utilizing MEDonline. Similar to normal lectures also each virtual lesson has a start date. For virtual lessons this is the date, from where the WBT parts of the virtual lessons are automatically generated within VMC/Moodle and presented to all students who are currently enrolled for the module where the virtual lesson is a part of it. When the virtual lesson ends results are transmitted back to MEDonline where a grade is automatically calculated. This process is visualized in Figure 5.

\section{Interface of Moodle to central administration system}

For the connection between VMC/Moodle and MEDonline a special interface (MOMOS - MEDonline interface) was developed in PHP [10] as an admin module for Moodle.

One of the main goals during the conceptual phase was preserving the current organizational workflow using MEDonline to plan courses and assign virtual lessons to specific dates as described in the last chapter.

All relevant MEDonline data could be retrieved through an existing CAMPUSonline Oracle schema called 
'CO LMS'. This schema includes several database views that are used e.g. to map user names to primary keys or get specific course data for a defined student. Another part of the schema is a stored procedure for saving exam results coming from external systems to database. The connection from MOMOS to Oracle is provided by the PHP OCI8 extension. For accessing the Moodle database standard Moodle library functions are used, providing independence from the underlying database (MySQL, PostgresSQL,...).

The admin modules task is to collect and process this MEDonline data to set the course startdate and visibility, the activity startdates and enddates (quiz, lesson and assignment), enroll and un-enroll students and send grades on a daily basis. The two main challenges for this were the conception of the actual business logic and the very poor Oracle connection performance under PHP. To avoid the slowdown by long Oracle connection times persistent connections were used and MOMOS functions and data queries were optimized for retrieving large junks of data minimize the number of Oracle requests. This approach proved to be reliable and fast. Each course can be tagged for processing by cronjobs automatically, or alternatively be excluded. In some cases manual processing is required for testing or correcting purposes. All actions can therefore be performed manually for individual courses or all courses as well. Filters provided for convenient access to active/inactive and courses selected for automatic processing. In order to provide safe and reliable automatic processing of $100+$ courses and several thousand students on a daily basis, two additional features have been added. All transactions are committed to daily plain text log files. The log files are human readable but also provide syntax for automatic error retrieval. The second feature is called "time machine". The MOMOS admin interface can be set to any date in the past or in the future and can execute all functions for the specified date. This feature is very useful for testing "what if" scenarios in the future or correcting errors e.g. resending grades for students that were unenrolled manually by accident in the past [8].

The MOMOS admin interface also provides an easy visualization of the system status. If all columns are highlighted in green all actions of the last cronjob were performed successfully. Errors are highlighted in red, warnings or status off in orange.

The "overview on my virtual mandatory tasks" is a modified version of the MyMoodle page that only displays courses linked with MOMOS. Due to the occasionally high number of courses (40 and more) the list is alphabetically sorted for better clarity.

\section{EXPERIENCES}

With the introduction of VMC/Moodle as the primary LMS on 1 Oct 2010124 virtual lessons were offered within VMC/Moodle containing all in all 258 WBTs (119 Moodle-quizzes und 139 Moodle-lessons). Within the winter semester 2010/11 1712 students enrolled in 12 different modules/tracks completed these virtual lessons and all in all 162,192 grades have already been submitted to MEDonline without any serious problems.

Due to the fact that virtual lessons must be executed by students within a specific time period in order to proceed with their study we had very high demands on VMC/Moodle in terms of stability, availability and per-

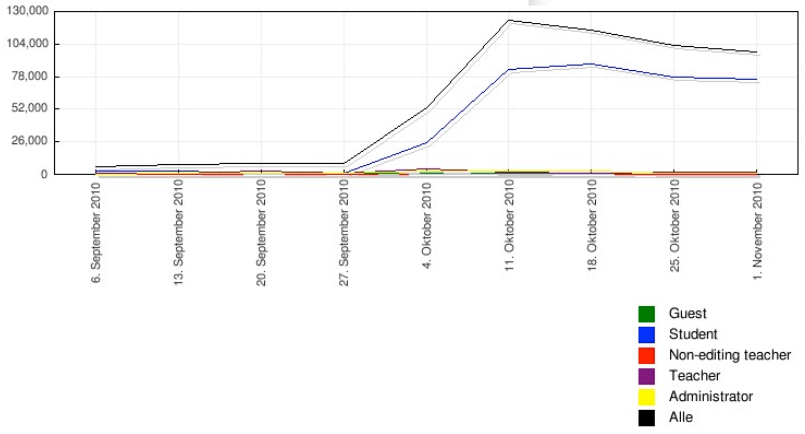

Figure 6. VMC/Moodle access statistics during the starting phase in Oct / Nov 2011

formance. As it can be seen in Figure 6. the monthly access rate from its start on 1 Oct 2010 is approximately 100,000 accesses a month, one peak reached even 130,000 accesses a month.

Nevertheless, the system neither seriously crashed since its start nor lacked from performance problems. Only once we had to perform a more extensive maintenance on the cluster configuration and for this purpose one of the servers had to be shut down. However, the active/active cluster configuration granted availability all over the time, and despite only one server of the cluster was online no performance problems could be discovered so far.

Since we did not have the specific Moodle know-how for implementing the MOMOS interface in house we decided to outsource it to a company [11] specialized in the implementation of Moodle modules and which also already had experiences with connecting Moodle to an external course administration system. However, the MOMOS interface was designed and implemented in close cooperation with a technical expert from the MUG and so we can now react very fast in case any unexpected behaviour appears in connection with the MOMOS interface and the virtual lessons. So far only minor issues appeared and could be solved by us without being dependent on the support of the external company.

\section{CONCLUSIONS}

We introduced the set-up of a highly available Moodle LMS and the technical implementation of fully automated virtual lessons within the LMS Moodle. In virtual lessons students are automatically enrolled and at the end grades are calculated based on tests students have to execute. So teachers can concentrate on continuously updating of the virtual content and can answer questions, which students might have during execution of the virtual lecture. Additionally, as most of our teachers are physicians, this process saves their valuable time resources, thus allowing more intensive bedside teaching.

The active/active cluster configuration of our VMC/Moodle LMS is according to our knowledge unique amongst all Moodle installations we know. We did the set up in close interdisciplinary co-operation with the MUGIT department, and performed several pilots. For the setup and maintenance technical in-house experts are absolutely necessary. The cluster turned out to be very stable and provides also enough performance even when one node goes down.

One important result of the evaluation of the Moodle pilot cluster was that Oracle in connection with a Moodle 
PAPER

The Set-Up and Implementation of Fully Virtualized Lessons with an Automated Workflow Utilizing VMC/Moodle at THE MEDICAL UnIVERSITY OF GRAZ

cluster configuration does not meet the performance requirements of the MUG. Even with only one active user response times were $>2$ seconds. We finally decided to use PostgreSQL [9] within the active / active cluster, which granted 3 to 6 times better response times than the Oracle solution.

\section{REFERENCES}

[1] Staber R, Smolle J, Bauer P, Hye F, Thallinger S, Neges H, Reibnegger G.: „Virtueller Medizinischer Campus Graz: eine eLearning Umgebung wird 5 Jahre alt“. GMS Med Bibl Inf. 2007; 7(3):Doc43. ISSN 1865-066X, <URL (22 June 2011): http://www.egms.de/en/journals/mbi/2007-7/mbi000095.shtml $>$

[2] Smolle J, Staber R, Hye F, Jamer E, Macher S, Neges H et al.: "ELearning in the First SEMESTER of an Undergraduate Medical Curriculum.”, 2006. EDEN conference proceedings. Ref Type: Electronic Citation Ref ID: 9210, <URL (22 June 2011): http://www.eden-online.org/contents/conferences/submit/upload/ Smolle.doc $>$

[3] Smolle J, Staber R, Jamer E, Reibnegger G.: „Aufbau eines universitätsweiten Lern- Informationssystems parallel zur Entwicklung innovativer Curricula - zeitliche Entwicklung und Synergieeffekte“. In: Tavangarian D, Nölting K, editors. Auf zu neuen Ufern - E-Learning heute und morgen. Münster New York München Berlin: Waxmann, 2005: 217-226. Ref ID: 9019

[4] H. Rehatschek, R. Staber, H. Neges, G. Reibnegger, J. Smolle: "The blended learning concept of the Medical University of Graz and its technical realization". In proceedings of the 13th Interactive Computer Aided Learning conference, pp. 54 - 59, ISBN 9783-89958-541-4, 15 - 17 September, 2010, Hasselt, Belgium.
[5] Moodle - Modular Object Oriented Dynamic Learning Environment, open source LMS, <URL (22 June 2011): http://www.moodle.org>

[6] Olat - Online Learning and Training, open source LMS of the ETH Zürich, 2011. <URL (22 June 2011): https://www.olat.uzh.ch/olat/dmz/>

[7] MEDonline is a specific implementation of CAMPUSonline ${ }^{\circledR}$ by Technical University of Graz, 2011. <URL (22 June 2011): http://online.tugraz.at >

[8] H. Rehatschek, G. Hölzl, A. Hruska: "The seamless integration of virtualized lessons with a fully automated workflow into the LMS VMC/Moodle of the Medical University of Graz". Online Proceedings of the MoodleMoot 2011 conference, 24 - 25 February 2011, Vienna, Austria

[9] PostgreSQL, 2011, open source object-relational database system. <URL (22 June 2011): http://www.postgresql.org>

[10] PHP - Hypertext Processor, 2011. <URL (26 June 2011): http://www.php.net/>

[11] eDaktik Hruska KG, 2010. <URL (26 June 2011): http://www.edaktik.com>

\section{AUTHORS}

Herwig Rehatschek, Gernot Hölzl, and Michael Fladischer are with Virtual Medical Campus, Medical University Graz, Graz, Austria. E-mail: \{Herwig.Rehatschek, Gernot.Hölzl, Michael.Fladischer\}@medunigraz.at.

Received 5 August 2011. Published as resubmitted by the authors 22 November 2011. 